\title{
Electric field induced structural colour tuning of a silver/titanium dioxide nanoparticle one-dimensional photonic crystal
}

\author{
Eduardo Aluicio-Sarduy ${ }^{1,2}$, Simone Callegari ${ }^{2}$, Diana Gisell Figueroa del Valle ${ }^{1,3}$, \\ Andrea Desii ${ }^{1}$, Ilka Kriegel ${ }^{4}$ and Francesco Scotognella ${ }^{* 1,3,5}$
}

Open Access

\author{
Full Research Paper \\ Address: \\ ${ }^{1}$ Center for Nano Science and Technology@PoliMi, Istituto Italiano di \\ Tecnologia, Via Giovanni Pascoli 70/3, 20133 Milan, Italy, \\ ${ }^{2}$ Dipartimento di Chimica, Materiali e Ingegneria Chimica "Giulio \\ Natta", Politecnico di Milano, Piazza Leonardo da Vinci 32, 20133 \\ Milano, Italy, ${ }^{3}$ Dipartimento di Fisica, Politecnico di Milano, Piazza \\ Leonardo da Vinci 32, 20133 Milano, Italy, ${ }^{4}$ Department of \\ Nanochemistry, Istituto Italiano di Tecnologia (IIT), via Morego 30, \\ 16163 Genova, Italy, and ${ }^{5}$ Istituto di Fotonica e Nanotecnologie CNR, \\ Piazza Leonardo da Vinci 32, 20133 Milano \\ Email: \\ Francesco Scotognella* - francesco.scotognella@polimi.it \\ * Corresponding author \\ Keywords: \\ electro-optic switching; photonic crystal; plasmonic nanoparticles
}

Beilstein J. Nanotechnol. 2016, 7, 1404-1410.

doi:10.3762/bjnano.7.131

Received: 24 May 2016

Accepted: 09 September 2016

Published: 06 October 2016

This article is part of the Thematic Series "Physics, chemistry and biology of functional nanostructures III".

Guest Editor: A. S. Sidorenko

(C) 2016 Aluicio-Sarduy et al.; licensee Beilstein-Institut. License and terms: see end of document.

\begin{abstract}
An electric field is employed for the active tuning of the structural colour in photonic crystals, which acts as an effective external stimulus with an impact on light transmission manipulation. In this work, we demonstrate structural colour in a photonic crystal device comprised of alternating layers of silver nanoparticles and titanium dioxide nanoparticles, exhibiting spectral shifts of around $10 \mathrm{~nm}$ for an applied voltage of only $10 \mathrm{~V}$. The accumulation of charge at the metal/dielectric interface with an applied electric field leads to an effective increase of the charges contributing to the plasma frequency in silver. This initiates a blue shift of the silver plasmon band with a simultaneous blue shift of the photonic band gap as a result of the change in the silver dielectric function (i.e. decrease of the effective refractive index). These results are the first demonstration of active colour tuning in silver/titanium dioxide nanoparticle-based photonic crystals and open the route to metal/dielectric-based photonic crystals as electro-optic switches.
\end{abstract}

\section{Introduction}

Structural colour is colour due to the Bragg reflection (in photonic structures for example) as opposed to colour from pigments or colour centres [1]. The active tuning of the structural colour in photonic crystals is a subject that has attracted a great attention in the last decades. The electric field is probably the simplest external stimulus that can be employed for such colour 
tuning. A recent review article has reported the most important achievements in the electrically driven tunability of photonic crystals [2]. In this interesting article, different types of tuning techniques are encompassed, for example: i) smart polymers [3-7], ii) liquid crystals [8-12], and electrophoresis [13-16].

The employment of metallic nanoparticles for the structural colour tuning with electric field, to the best of our knowledge, has not been reported in the literature. However, plasmon peak tuning of gold nanoparticles with an electric field in an electrochemical cell has been recently shown [17], opening the way to a new strategy for electro-optical switches with metal nanostructures.

In this paper we show experimental evidence of structural colour tuning with an electric field in a one-dimensional photonic crystal made of alternating layers of silver nanoparticles and titanium dioxide nanoparticles. We have observed a blue shift of about $10 \mathrm{~nm}$ with an applied voltage of $10 \mathrm{~V}$. We give an interpretation of the phenomenon based on the increased carrier density participating in the plasma frequency of silver. Such charges are due to the polarization at the titanium dioxide/silver interface upon application of an electric field.

\section{Results and Discussion}

The fabricated photonic crystal is made of five bilayers of silver nanoparticles and titanium dioxide nanoparticles deposited on top of an indium tin oxide (ITO) substrate. A scheme of the photonic crystal is shown in Figure 1.

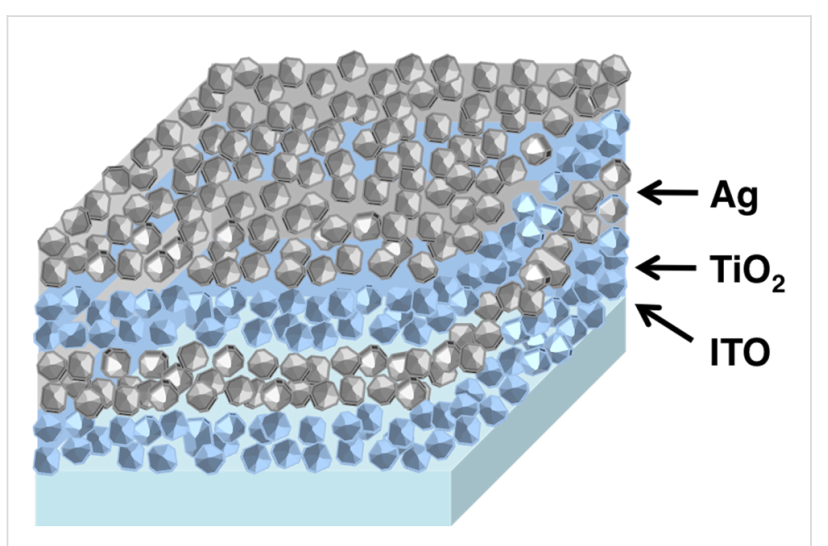

Figure 1: Scheme of the one-dimensional photonic crystal made of layers of silver nanoparticles and titanium dioxide nanoparticles. The actual photonic crystal fabricated in this work is composed of five silver/titanium dioxide bilayers.

The silver nanoparticles have a diameter of about $50 \mathrm{~nm}$, while the $\mathrm{TiO}_{2}$ nanoparticles are smaller than $15 \mathrm{~nm}$. The surface topography and phase atomic force microscopy (AFM) images of a $\mathrm{Ag}$ layer, a $\mathrm{TiO}_{2}$ layer and the top surface of a five bilayer
$\mathrm{Ag} / \mathrm{TiO}_{2}$ photonic crystal, all deposited on glass/ITO substrates, are presented in the Supporting Information File 1 (Figure S1) and show that the silver nanoparticle layer has the highest surface roughness among the different samples. This is due to the large size of the silver nanoparticles and the formation of large aggregates. Notably, the lowest surface roughness was found for the top $\mathrm{TiO}_{2}$ surface layer of the five bilayer photonic crystal (Figure S1c of Supporting Information File 1). The formation of a more compact layer with a reduced surface roughness is most probably a result of the small $\mathrm{TiO}_{2}$ nanoparticles deposited onto the Ag films, which fill the empty voids between the Ag aggregates and promote a certain degree of intermixing between the two different types of nanoparticles at the $\mathrm{Ag} / \mathrm{TiO}_{2}$ interfaces. Indeed, scanning electron microscopy (SEM) images (Figure S2 in Supporting Information File 1) of the cross section of a photonic crystal shows five bilayers (the single silver and $\mathrm{TiO}_{2}$ layers cannot be distinguished due to the resolution of our instrument) of a total thickness of $600 \mathrm{~nm}$. This value is lower than the sum of five single $\mathrm{Ag}$ and $\mathrm{TiO}_{2}$ layers deposited directly on the substrate $(60 \mathrm{~nm}$ and $120 \mathrm{~nm}$, respectively), confirming the conclusions of the AFM analysis. Nevertheless, we consider that this intermixing is confined to the interface region and that a bilayer structure is still obtained with a blurred interface.

For the electro-optical characterization, we placed another ITO substrate on the other side of the photonic crystal and applied an external voltage to provide an electric field to the photonic crystal device. The electro-optical measurement is shown in Figure 2, where the transmission spectrum of the photonic crystal is reported as a function of the applied voltage. The transmission is dominated by two strong bands at around $480 \mathrm{~nm}$ and $620 \mathrm{~nm}$, ascribed to the plasmonic resonances of the silver nanoparticles and the photonic bandgap, respectively.

We want to emphasize the fundamentally different nature of the two resonances observed in our device, namely the plasmonic resonance of the silver nanoparticle layer and that of the photonic bandgap. The pump-probe measurement in Figure $3 \mathrm{a}$ shows the transmission spectra of the transient absorption measurements at delay times of 500 and 3000 fs (black and red curve, respectively). We observe the typical plasmonic response of the silver nanoparticles as a derivative shape of the peak at $480 \mathrm{~nm}$ (Figure 3a). The temporal behaviour of metallic nanoparticles is characterized by three different regimes [18]: i) the pump excitation strongly perturbs the Fermi distribution and creates electrons that are not in thermal equilibrium, called energetic electrons; via electron-electron scattering, within a few tens to hundreds of fs, a new Fermi distribution of hot electrons is obtained. ii) Within a few ps, the hot electrons release their energy to the lattice via electron-phonon scattering. 


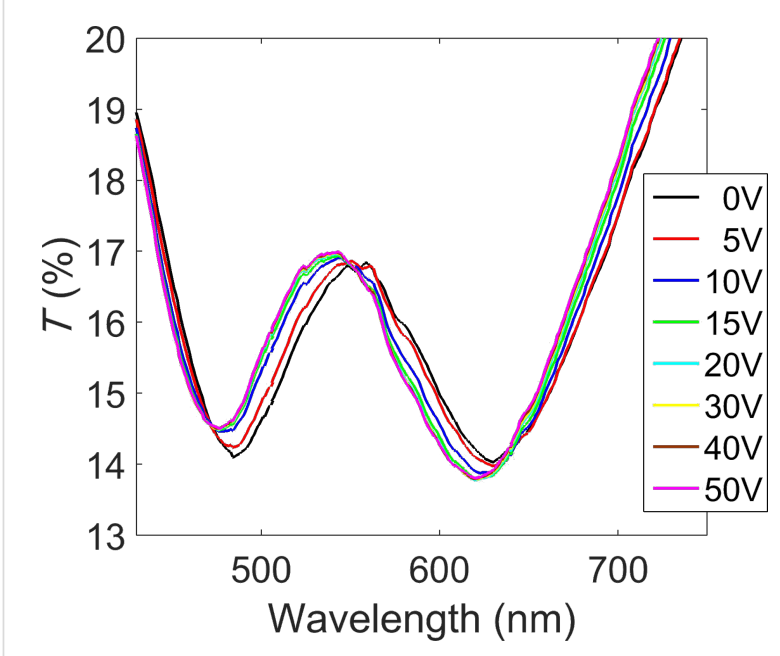

Figure 2: Transmission spectra of the ITO-(Ag nanoparticle/TiO nanoparticle $)_{5}-$ ITO photonic crystal device upon application of an electric field.

iii) The hot lattice releases its energy to the environment within hundreds of ps. With the temporal resolution of our setup, which is about $150 \mathrm{fs}$, we could not observe the electron-electron scattering, but the dynamic of the resonance at $450 \mathrm{~nm}$ reported in Figure $3 \mathrm{~b}$ is related to the electron-phonon scattering. This picosecond-scale dynamic is followed by a very weak phonon-phonon scattering. The photonic band gap (around $620 \mathrm{~nm}$ ) does not show any particular dynamic (not shown here), as expected. The combination of a metal and a dielectric in the photonic device is a key to the voltage-dependent observations, as will be explained later in this manuscript.

Upon application of an electrical potential to the device, we observe a blue shift of the entire transmission spectrum, that is, of the photonic band gap as well as the plasmon resonance of the silver nanoparticles. The shift of the photonic band gap is about $10 \mathrm{~nm}$ for an applied potential of only $10 \mathrm{~V}$. In Figure S7 of Supporting Information File 1 we show that at voltages above $15 \mathrm{~V}$ the shift saturates up to a value of about 16-17 nm. Notably, the observed shifts of both resonances are a result of the alternation between the metal and the dielectric nanoparticle layer, as affirmed by several counter experiments, as demonstrated in Figure 4.

We investigated three scenarios: first, the results for devices with only ITO substrates placed together, as given in Figure 4a; second, the results of only the silver layer between two ITO substrates, as given in Figure $4 b$; and third, for only the titanium dioxide layer between two ITO substrates (Figure 4c). For all three investigated cases, the observed spectral changes by applying a potential to the device are negligible, even in the region of the silver plasmon band (Figure $4 \mathrm{~b}$ ). The strong red

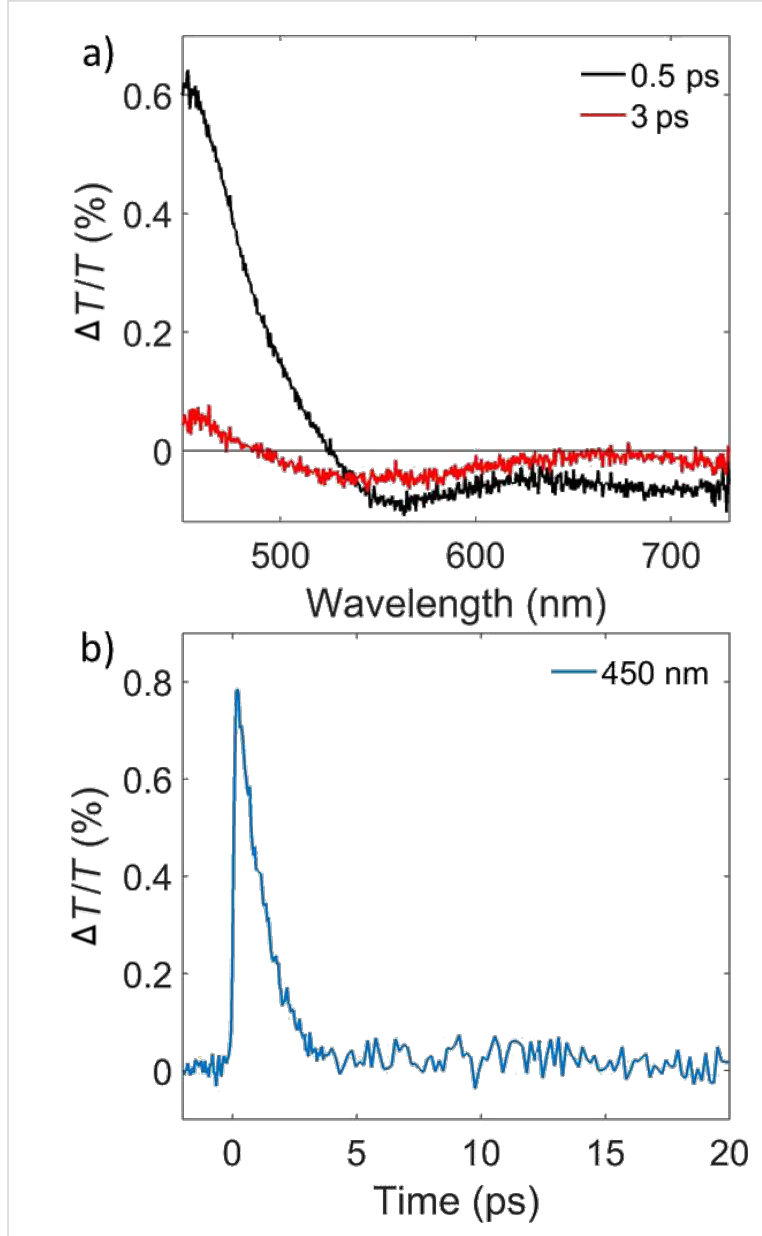

Figure 3: (a) Pump-probe spectra at different time delays and (b) pump-probe dynamic at $450 \mathrm{~nm}$ of the ITO-(Ag nanoparticle/ $/ \mathrm{TiO}_{2}$ nanoparticle) $)_{5}$-ITO photonic crystal.

shift of the plasmonic peak when the silver nanoparticle layer is deposited on ITO $(620 \mathrm{~nm})$ with respect to the glass substrate $(480 \mathrm{~nm})$ was observed in the static samples (i.e. without applying the voltage is ascribed to a coupling between the high carrier density of ITO and the silver nanoparticle plasmon). A difference in fill factor might also play a role here, leading to a stronger coupling and an intense red shift. Nevertheless, these results demonstrate that the observed shifts with applied voltage are only observed in the alternating silver and titanium dioxide nanoparticle layers.

In the following we provide an interpretation for the observed blue shift of the photonic band gap as well as the silver plasmon resonance by applying an electric field and making a simple assumption. We consider the plasma frequency $\omega_{\mathrm{p}}$ for silver as

$$
\omega_{\mathrm{p}}=\sqrt{\frac{N e^{2}}{m^{*} \varepsilon_{0}}},
$$



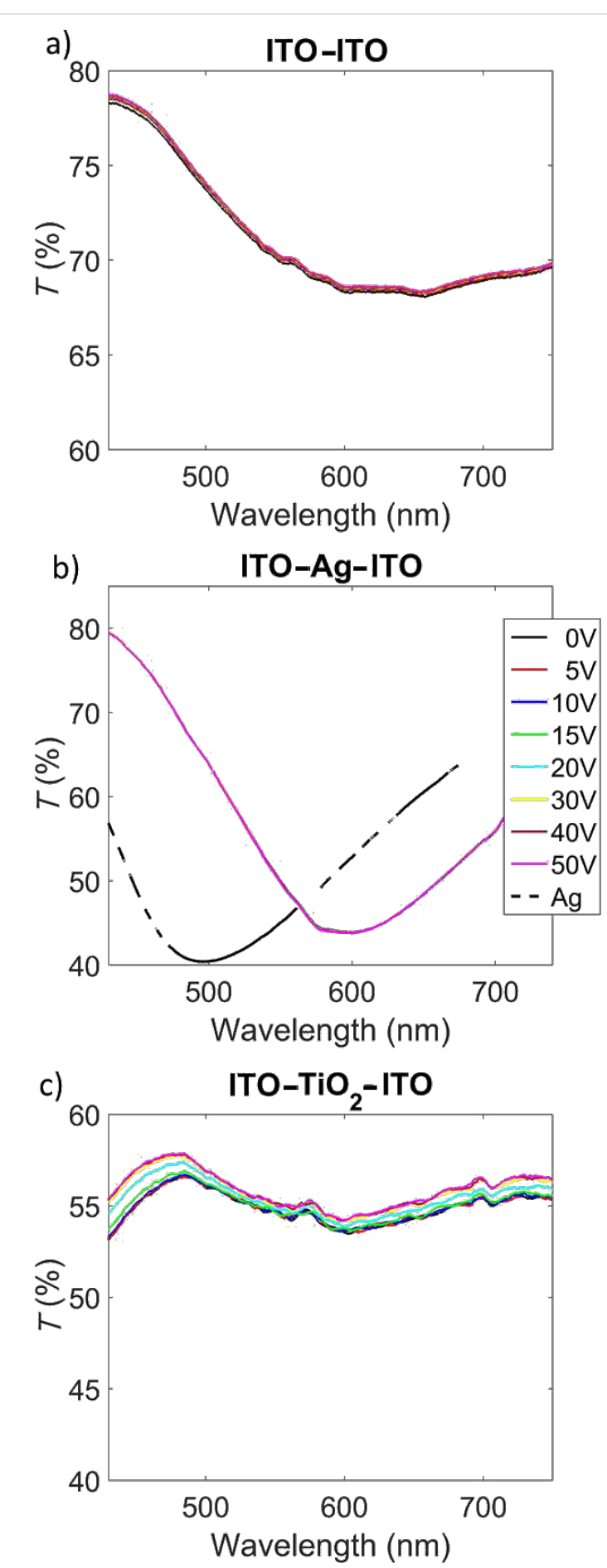

Figure 4: (a) ITO-ITO device under electric field; (b) ITO-Ag nanoparticle-ITO device under electric field; (c) ITO- $\mathrm{TiO}_{2}$ nanoparticle-ITO device under electric field.

where $N$ is the carrier density, $e$ is the electron charge, $m *$ is the effective mass and $\varepsilon_{0}$ is the dielectric constant of the vacuum. Qualitatively, we can state that the polarization charges that accumulate at the silver/titanium dioxide interface, because of the electric field, effectively increase the carrier density

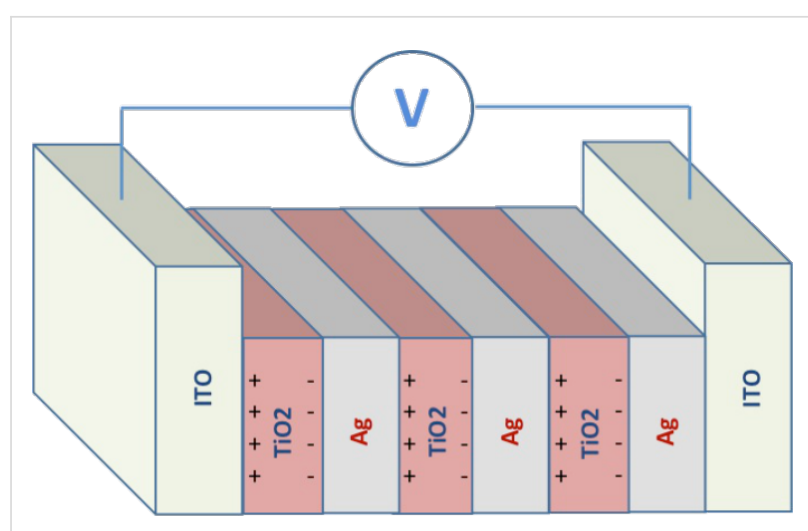

Figure 5: Scheme of the interpretation of the action of the electric field on the ITO-(Ag nanoparticle $/ \mathrm{TiO}_{2}$ nanoparticle $)_{5}-\mathrm{Ag}-\mathrm{ITO}$ photonic crystal device.

involved in the plasma frequency, as schematically depicted in Figure 5. Moreover, a second contribution can be the flow of charges in the silver layers due to the electric field itself. In this way, we have the carrier density with the electric field $\left(N^{\mathrm{E}}\right)$ versus the initial carrier density $(N)$, such that $N^{\mathrm{E}}>N$.

The Drude model can be used to predict the behaviour of the plasmonic response in the photonic crystal [19]. The frequencydependent complex dielectric function of silver can be written as

$$
\varepsilon_{\mathrm{Ag}}(\omega)=\varepsilon_{1}(\omega)+\mathrm{i} \varepsilon_{2}(\omega)
$$

where

$$
\varepsilon_{1}=\varepsilon_{\infty}-\frac{\omega_{p}^{2}}{\left(\omega^{2}-\Gamma^{2}\right)}
$$

and

$$
\varepsilon_{2}=\frac{\omega_{\mathrm{p}}^{2} \Gamma}{\omega\left(\omega^{2}-\Gamma^{2}\right)}
$$

with $\Gamma$ representing the free carrier damping [20].

The dielectric function of the silver nanoparticle film (a network of necked silver nanoparticles with air pores) can be described by the Maxwell-Garnett effective medium approximation [21-23], which is given by

$$
\varepsilon_{\text {eff,Ag }}=\varepsilon_{\text {Air }} \frac{2\left(1-\delta_{\mathrm{Ag}}\right) \varepsilon_{\mathrm{Air}}+\left(1+2 \delta_{\mathrm{Ag}}\right) \varepsilon_{\mathrm{Ag}}}{2\left(2+\delta_{\mathrm{Ag}}\right) \varepsilon_{\mathrm{Air}}+\left(1-\delta_{\mathrm{Ag}}\right) \varepsilon_{\mathrm{Ag}}},
$$


where $\varepsilon_{\text {Air }}$ is the dielectric constant of air, and $\delta_{\mathrm{Ag}}$ accounts for the volume fraction occupied by the silver nanoparticles. Given $n_{\text {eff, } \mathrm{Ag}^{2}}=\varepsilon_{\text {eff,Ag }}$ and taking the refractive index dispersion of titanium dioxide from the literature $[24,25]$ we used the transfer matrix method $[26,27]$ to simulate the transmission spectrum of the photonic crystal. We remark here that the dielectric function of both the $\mathrm{Ag}$ and the $\mathrm{TiO}_{2}$ nanoparticle layers were calculated with the effective medium approximation, as is thoroughly discussed in Supporting Information File 1. In Figure 6 the results of the calculation for three different carrier densities are given, where blue is the actual carrier density of $\mathrm{Ag}$ as given in [28] and two artificially increased carrier densities. Similar to the experimental results, the calculated transmission spectra show an intense band in the UV/blue region ascribed to the plasmon resonance of the silver layer and a second band corresponding to the photonic bandgap. Note that the plasmon resonance of silver overlaps with some thin film interference features (see Figure S4 in the Supporting Information File 1). To unambiguously assign the plasmon resonance, we performed simulations to distinguish the absorption contribution (i.e. the imaginary part of the refractive index of Ag, see Figure S5 in the Supporting Information File 1). From the simulation of the spectra, we see that an increase of the carrier density induces a blue shift of the photonic band gap, confirming the interpretation of our experimental findings.

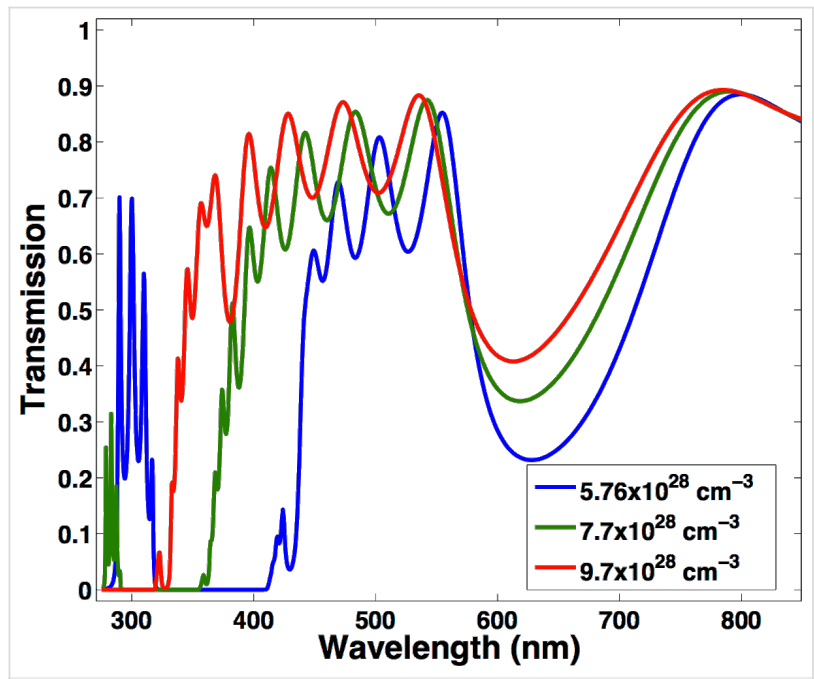

Figure 6: Transmission spectra simulated with the transfer matrix method for a $\mathrm{Ag} / \mathrm{TiO}_{2}$ nanoparticle photonic crystal device with three different carrier densities.

We used the same model to estimate the number of charges added to the system. Without the applied voltage (Supporting Information File 1, Figure S3), we used the parameters for the silver dielectric function as reported in [28] with the electron density of silver $N=5.76 \times 10^{28} \mathrm{~m}^{-3}$ to simulate the optical properties of the multilayer structure. To get an estimate of the number of charges introduced with an applied voltage of $40 \mathrm{~V}$ (Supporting Information File 1, Figure S6), we had to increase the carrier density to the value of $N^{\mathrm{E}}=6.86 \times 10^{28} \mathrm{~m}^{-3}$. This resulted in a difference of $\Delta N=1.1 \times 10^{28} \mathrm{~m}^{-3}$. Taking into account that the five silver layers have a volume of $\left(60 \times 10^{-9} \mathrm{~m} \times 5\right) \times 0.015 \mathrm{~m} \times 0.015 \mathrm{~m}$, and that the diameter of the Ag nanoparticles is about $50 \mathrm{~nm}$, we could estimate an increase of about $10^{5}$ charges per particle. We remark here that this discussion just gives a rough estimate of the required number of charges added to each nanoparticle to induce the shifts observed. Indeed, in the model, we assumed for simplicity a change in the carrier density over the entire volume of the nanocrystal, although, as studied in [17], charge accumulation in metallic nanoparticles occurs for diameters around $5 \mathrm{~nm}$ only (in contrast to the $50 \mathrm{~nm}$ diameter). An exact evaluation would require a deeper analysis. Moreover, Brown et al. [17] showed that the electrochemical doping of gold nanoparticles in solution is, apart from a change in carrier density, accompanied by an increase in the surrounding medium refractive index. A change of the dielectric surrounding, however, also largely influences the position of the plasmon resonance [29]. In addition, doping occurs only in a thin layer at the nanoparticle surface. These two effects also ultimately impact on the carrier damping, which in our estimation was kept constant. Studies on the accumulation of charges in an ITO film by applying a constant voltage demonstrated that besides an increase in carrier density, other Drude parameters such as the damping constant and the high frequency dielectric constant are altered through the introduction of additional carriers [30]. Thus, a deeper study of the effect of applied voltage on thin films of Ag would be required for an exact evaluation of the effect on the Drude parameters and a more precise extraction of the number of carriers injected. Nevertheless, our estimation is in good agreement with results on the electrochemical doping of Au nanoparticles in solution, as observed by Ung et al [31]. Here the injection of $1600 \pm 300$ carriers was found in nanoparticles of $11.5 \mathrm{~nm}$ in diameter, which corresponds to an increase in the carrier density by around $\Delta N=2 \times 10^{27} \mathrm{~m}^{-3}$. This value is about one order of magnitude below our findings, corresponding to the order of magnitude lower carrier density of Au with respect to Ag.

\section{Conclusion}

In this work we studied the tuning of the structural colour, that is, the active shift of the photonic band gap, in a one-dimensional photonic crystal made of alternating silver nanoparticle and titanium dioxide nanoparticle layers. A concomitant blue shift of the silver plasmon peak and of the photonic band gap of about $10 \mathrm{~nm}$ with a $10 \mathrm{~V}$ applied voltage was observed. We have proposed an interpretation of this observation in this article: the electric field induces the accumulation of polarization charges at the silver/titanium dioxide interface. These 
charges contribute to the plasma frequency of silver, which due to the porosity of the layer and the subsequent high surface/ volume ratio, will allow the electron density to increase over the entire volume of the silver layer, resulting in an increase of the carrier density and a blue shift of the plasma frequency. We estimated an increase in carrier density by $\Delta N=1.1 \times 10^{28} \mathrm{~m}^{-3}$. Consequently, the effective refractive index of the whole photonic crystal is also changed, leading to the blue shift of the photonic band gap. Our results highlight the possibility to employ these photonic structures to manipulate the transmission of light.

\section{Methods}

\section{Nanoparticle colloidal dispersions}

Silver nanoparticle dispersion was purchased by SigmaAldrich and was diluted in triethylene glycol monoethyl ether (Sigma-Aldrich) up a final concentration of $5 \mathrm{wt} \%$. The diameter of the nanoparticles was less than $50 \mathrm{~nm}$. The $\mathrm{TiO}_{2}$ sol was synthesized by following a protocol reported in the literature based on the hydrolysis of titanium tetraisopropoxide $\left(\mathrm{Ti}\left(\mathrm{OCH}_{2} \mathrm{CH}_{2} \mathrm{CH}_{3}\right)_{4}\right.$ (TTIP, $97 \%$, purchased from Sigma-Aldrich) [32]. Briefly, a mixture of $2.5 \mathrm{~mL}$ of ethanol and $15 \mathrm{~mL}$ of TTIP was added dropwise, in a three-neck round bottom flask, to $90 \mathrm{~mL}$ of distilled water to obtain a TTIP/ethanol/water mixture with a molar ratio of 1:0.75:83. Subsequently, $1 \mathrm{~mL}$ of hydrochloric acid (purchased by SigmaAldrich) was added and the obtained sol was refluxed under stirring for $8 \mathrm{~h}$ at $80{ }^{\circ} \mathrm{C}$, resulting in a stable, milky solution. Before layer deposition, we concentrated the nanoparticle dispersion in order to make thicker layers.

\section{Photonic crystal fabrication}

The photonic crystal was fabricated on an indium tin oxide (ITO) substrate using a spin coater (Laurell, WS-400- 6NPPLite). The rotation speeds for the deposition were $2000 \mathrm{rpm}$ and $2000 \mathrm{rpm}$ for silver and titanium dioxide nanoparticles, respectively. After each deposition, the sample was annealed for $10 \mathrm{~min}$ at $350{ }^{\circ} \mathrm{C}$ on a hot plate under the fume hood.

\section{Optical measurements with electric field}

The photonic crystal, fabricated on ITO substrate, was covered with another ITO substrate in order to apply an electric field. To apply an electric field, we employed a simple voltage supply with a $100 \times$ amplifier. The transmission spectra were collected with a Shimazdu spectrophotometer.

\section{Pump-probe experiment}

For this experiment, an amplified Ti:sapphire laser system was employed (150 fs pulse duration, $1 \mathrm{kHz}$ repetition rate, $800 \mathrm{~nm}$ excitation wavelength). The pump pulse at $400 \mathrm{~nm}$ was achieved via second harmonic generation. The light transmis- sion was probed with broadband supercontinuum generation in sapphire. The signal was collected by a fast CCD camera connected to a spectrometer and was presented as the differential transmission $\Delta \mathrm{T} / \mathrm{T}[33]$.

\section{Supporting Information}

\section{Supporting Information File 1}

Morphological characterization of the sample with atomic force microscopy and scanning electron microscopy; short description of the transfer matrix method; additional simulations of the light transmission in bare silver films and in the photonic crystal.

[http://www.beilstein-journals.org/bjnano/content/ supplementary/2190-4286-7-131-S1.pdf]

\section{References}

1. Vignolini, S.; Rudall, P. J.; Rowland, A. V.; Reed, A.; Moyroud, E.; Faden, R. B.; Baumberg, J. J.; Glover, B. J.; Steiner, U. Proc. Natl. Acad. Sci. U. S. A. 2012, 109, 15712-15715. doi:10.1073/pnas.1210105109

2. Nucara, L.; Greco, F.; Mattoli, V. J. Mater. Chem. C 2015, 3, 8449-8467. doi:10.1039/C5TC00773A

3. Arsenault, A. C.; Puzzo, D. P.; Manners, I.; Ozin, G. A. Nat. Photonics 2007, 1, 468-472. doi:10.1038/nphoton.2007.140

4. Lu, Y.; Xia, H.; Zhang, G.; Wu, C. J. Mater. Chem. 2009, 19, 5952. doi:10.1039/b905760a

5. Lu, Y.; Meng, C.; Xia, H.; Zhang, G.; Wu, C. J. Mater. Chem. C 2013, 1, 6107. doi:10.1039/c2tc00747a

6. Norton, J.; Han, M. G.; Jiang, P.; Creager, S.; Foulger, S. H. J. Mater. Chem. 2007, 17, 1149-1153. doi:10.1039/b618348d

7. Ueno, K.; Matsubara, K.; Watanabe, M.; Takeoka, Y. Adv. Mater. 2007, 19, 2807-2812. doi:10.1002/adma.200700159

8. Shimoda, Y.; Ozaki, M.; Yoshino, K. Appl. Phys. Lett. 2001, 79, 3627. doi:10.1063/1.1421080

9. Ozaki, M.; Shimoda, Y.; Kasano, M.; Yoshino, K. Adv. Mater. 2002, 14, 514-518.

doi:10.1002/1521-4095(20020404)14:7<514::AID-ADMA514>3.0.CO;2 $-4$

10. Kubo, S.; Gu, Z.-Z.; Takahashi, K.; Fujishima, A.; Segawa, H.; Sato, O. Chem. Mater. 2005, 17, 2298-2309. doi:10.1021/cm050249।

11. Criante, L.; Scotognella, F. J. Phys. Chem. C 2012, 116, 21572-21576. doi:10.1021/jp309061r

12. Criante, L.; Scotognella, F. Mol. Cryst. Liq. Cryst. 2013, 572, 31-39. doi:10.1080/15421406.2012.763207

13. Shim, T. S.; Kim, S.-H.; Sim, J. Y.; Lim, J.-M.; Yang, S.-M. Adv. Mater. 2010, 22, 4494-4498. doi:10.1002/adma.201001227

14. Han, M. G.; Shin, C. G.; Jeon, S.-J.; Shim, H.; Heo, C.-J.; Jin, H.; Kim, J. W.; Lee, S. Adv. Mater. 2012, 24, 6438-6444. doi:10.1002/adma.201203211

15. Han, M. G.; Heo, C.-J.; Shin, C. G.; Shim, H.; Kim, J. W.; Jin, Y. W.; Lee, S. J. Mater. Chem. C 2013, 1, 5791. doi:10.1039/c3tc31192a

16. Luo, Y.; Zhang, J.; Sun, A.; Chu, C.; Zhou, S.; Guo, J.; Chen, T.; Xu, G. J. Mater. Chem. C 2014, 2, 1990. doi:10.1039/c3tc32227k 
17. Brown, A. M.; Sheldon, M. T.; Atwater, H. A. ACS Photonics 2015, 2, 459-464. doi:10.1021/ph500358q

18. Del Fatti, N.; Voisin, C.; Achermann, M.; Tzortzakis, S.; Christofilos, D.; Vallée, F. Phys. Rev. B 2000, 61, 16956-16966. doi:10.1103/PhysRevB.61.16956

19. Müller, J.; Sönnichsen, C.; von Poschinger, H.; von Plessen, G.; Klar, T. A.; Feldmann, J. Appl. Phys. Lett. 2002, 81, 171. doi:10.1063/1.1491003

20. Novotny, L.; Hecht, B. Principles of nano-optics, 2nd ed.; Cambridge University Press: Cambridge, United Kingdom, 2012. doi:10.1017/CBO9780511794193

21. Li, S.-Y.; Niklasson, G. A.; Granqvist, C. G. Appl. Phys. Lett. 2012, 101 , 71903. doi:10.1063/1.4739792

22. Mendelsberg, R. J.; Garcia, G.; Li, H.; Manna, L.; Milliron, D. J. J. Phys. Chem. C 2012, 116, 12226-12231. doi:10.1021/jp302732s

23. Mendelsberg, R. J.; Garcia, G.; Milliron, D. J. J. Appl. Phys. 2012, 111, 063515. doi:10.1063/1.3695996

24. Hasan, M. M.; Haseeb, A. S. M. A.; Saidur, R.; Masjuki, H. H. World Acad. Sci., Eng. Technol. 2008, 2, 221-225.

25. Scotognella, F.; Chiasera, A.; Criante, L.; Aluicio-Sarduy, E.; Varas, S.; Pelli, S.; Łukowiak, A.; Righini, G. C.; Ramponi, R.; Ferrari, M. Ceram. Int. 2015, 41, 8655-8659. doi:10.1016/j.ceramint.2015.03.077

26. Born, M.; Wolf, E. Principles of Optics: Electromagnetic Theory of Propagation, Interference and Diffraction of Light; CUP Archive, 2000.

27. Bendickson, J. M.; Dowling, J. P.; Scalora, M. Phys. Rev. E 1996, 53, 4107-4121. doi:10.1103/PhysRevE.53.4107

28. Electrical Resistivity, Thermoelectrical Power and Optical Properties; Hellwege, K.-H.; Olsen, J. L., Eds.; Landolt-Börnstein - Group III Condensed Matter, Vol. 15b; Springer: Berlin, Germany, 1985. doi:10.1007/b19992

29. Mulvaney, P. Langmuir 1996, 12, 788-800. doi:10.1021/la9502711

30. Feigenbaum, E.; Diest, K.; Atwater, H. A. Nano Lett. 2010, 10 , 2111-2116. doi:10.1021/nl1006307

31. Ung, T.; Giersig, M.; Dunstan, D.; Mulvaney, P. Langmuir 1997, 13, 1773-1782. doi:10.1021/la960863z

32. Yun, Y. J.; Chung, J. S.; Kim, S.; Hahn, S. H.; Kim, E. J. Mater. Lett. 2004, 58, 3703-3706. doi:10.1016/j.matlet.2004.07.018

33. Cerullo, G.; Manzoni, C.; Lüer, L.; Polli, D. Photochem. Photobiol. Sci. 2007, 6, 135-144. doi:10.1039/B606949E

\section{License and Terms}

This is an Open Access article under the terms of the Creative Commons Attribution License (http://creativecommons.org/licenses/by/4.0), which permits unrestricted use, distribution, and reproduction in any medium, provided the original work is properly cited.

The license is subject to the Beilstein Journal of Nanotechnology terms and conditions: (http://www.beilstein-journals.org/bjnano)

The definitive version of this article is the electronic one which can be found at: doi:10.3762/bjnano.7.131 